\title{
Camera of the first TAIGA-IACT: construction and calibration
}

N. Lubsandorzhiev ${ }^{* 1}$, I. Astapov ${ }^{9}$, P. Bezyazeekov², V. Boreyko ${ }^{10}$, A. Borodin ${ }^{10}$, M. Brueckner ${ }^{8}$, N. Budnev², A. Chiavassa ${ }^{4}$, A. Dyachok ${ }^{2}$, O. Fedorov ${ }^{2}$, A. Gafarov², A. Garmash ${ }^{11}$, N. Gorbunov ${ }^{10,14}$, V. Grebenyuk ${ }^{10,14}$, O. Gress ${ }^{2}$, T. Gress ${ }^{2}$, O. Grishin ${ }^{2}$, A. Grinyuk ${ }^{10}$, D. Horns ${ }^{6}$, A. Igoshin ${ }^{1}$, A. Ivanova ${ }^{2}$, N. Kalmykov ${ }^{1}$, Y. Kazarina ${ }^{2}$, V. Kindin ${ }^{9}$, P. Kirilenko ${ }^{11}$, S. Kiryuhin ${ }^{2}$, R. Kokoulin ${ }^{9}$, K. Kompaniets ${ }^{9}$, E. Korosteleva ${ }^{1}$, V. Kozhin ${ }^{1}$, E. Kravchenko ${ }^{11,12}$, M. Kunnas ${ }^{6}$, L. Kuzmichev¹,2, Yu. Lemeshev ${ }^{2}$, V. Lenok ${ }^{2}$, B. Lubsandorzhiev ${ }^{1,3}$, R. Mirgazov $^{2}$, R. Mirzoyan ${ }^{5,2}$, R. Monkhoev ${ }^{2}$, R. Nachtigall ${ }^{6}$, E. Osipova ${ }^{2}$, A. Pakhorukov ${ }^{2}$, M. Panasyuk ${ }^{1}$, L. Pankov², A. Petrukhin ${ }^{9}$, V. Poleschuk², E. Popescu ${ }^{13}$, E. Popova ${ }^{1}$, A. Porelli $^{8}$, E. Postnikov $^{1}$, V. Prosin', V. Ptuskin, E. Rjabov², G. Rubtsov³, A. Pushnin², Y. Sagan $^{10}$, B. Sabirov ${ }^{10}$, V. Samoliga ${ }^{2}$, Yu. Semeney ${ }^{2}$, A.Silaev $^{1}$, A. Silaev (junior) ${ }^{1}$, A. Sidorenkov ${ }^{3}$, A. Skurikhin ${ }^{1}$, V. Slunecka ${ }^{10}$, A. Sokolov ${ }^{11,12}$, C. Spiering ${ }^{8}$,

L. Sveshnikova ${ }^{1}$, V. Tabolenko ${ }^{2}$, B. Tarashansky ${ }^{2}$, A. Tkachenko ${ }^{10}$,

L. Tkachev ${ }^{10,14}$, M. Tluczykont ${ }^{6}$, R. Wischnewski ${ }^{8}$, A. Zagorodnikov ${ }^{2}$, D. Zhurov ${ }^{2}$, V. Zurbanov' ${ }^{2}$ I. Yashin ${ }^{9}$

${ }^{1}$ Skobeltsyn Institute of Nuclear Physics MSU, Moscow, Russia, ${ }^{2}$ Institute of Applied Physics ISU, Irkutsk, Russia, ${ }^{3}$ Institute for Nuclear Research RAS, Moscow, Russia, ${ }^{4}$ Dipartimento di Fisica Generale Universiteta di Torino and INFN, Torino, Italy, ${ }^{5}$ Max-Planck-Institute for Physics, Munich, Germany, ${ }^{6}$ Institute for Experimental Physics, University of Hamburg, Germany, ${ }^{7}$ IZMIRAN, Moscow, Russia, ${ }^{8}$ DESY, Zeuthen, Germany, ${ }^{9}$ National Research Nuclear University MEPhI (Moscow Engineering Physics Institute), Moscow, Russia, ${ }^{10} \mathrm{Joint}$ Institute for Nuclear Research, Dubna, Russia, ${ }^{11}$ Novosibirsk State University, NSU, Novosibirsk, Russia, ${ }^{12}$ Budker Institute of Nuclear Physics SB RAS, Novosibirsk, Russia, ${ }^{13} I S S$, Bucharest, Romania, ${ }^{14}$ Dubna State University, Dubna, Russia E-mail: nilubs@rambler.ru

\begin{abstract}
The first imaging atmospheric Cherenkov telescope as a part of the TAIGA (Tunka Advanced Instrument for cosmic rays and Gamma Astronomy) detector was put into commission in the Tunka valley in December 2016. The reflector of the telescope follows the Davis-Cotton design. It is composed of 34 glassmirror tiles, each of $60 \mathrm{~cm}$ diameter, and has a diameter of $4.3 \mathrm{~m}$. The focal length is $4.75 \mathrm{~m}$. The imaging camera includes 560 photomultiplier-based pixels, providing a FoV of $\sim 10 \times 10$ degrees. The imaging camera is made by using a modular principle and includes trigger, data acquisition and processing systems based on MAROC3 chips. The slow control system monitors the count rate and the PMT anode currents as well as controls the high voltage. For monitoring performance of the camera a dedicated calibration system based on nanosecond pulsed LED light source was developed.
\end{abstract}

35th International Cosmic Ray Conference - ICRC2017

10-20 July, 2017

Bexco, Busan, Korea

${ }^{*}$ Speaker 


\section{Introduction}

The first imaging atmospheric Cherenkov telescope of the TAIGA project [1, 2] has started commissioning in December 2016 (figure 1). The TAIGA-IACT array of imaging telescopes is under development for joint detection of gamma-rays at energies above $30 \mathrm{TeV}$ together with the TAIGA-HiSCORE timing array of wide-angle atmospheric Cherenkov stations [3, 4]. With such an approach EAS parameters (arrival direction, core position, energy of primary particle and maximum of shower development) will be reconstructed by the non-imaging timing array while gamma-hadron separation will be done by the imaging telescope. Thus the TAIGA-IACT telescopes can operate in non-stereoscopic mode that allows to build an array of imaging telescopes covering a large area of up to $5 \mathrm{~km}^{2}$ but with a relatively small number of telescopes.

This report presents description of the TAIGA-IACT camera and some calibration results.

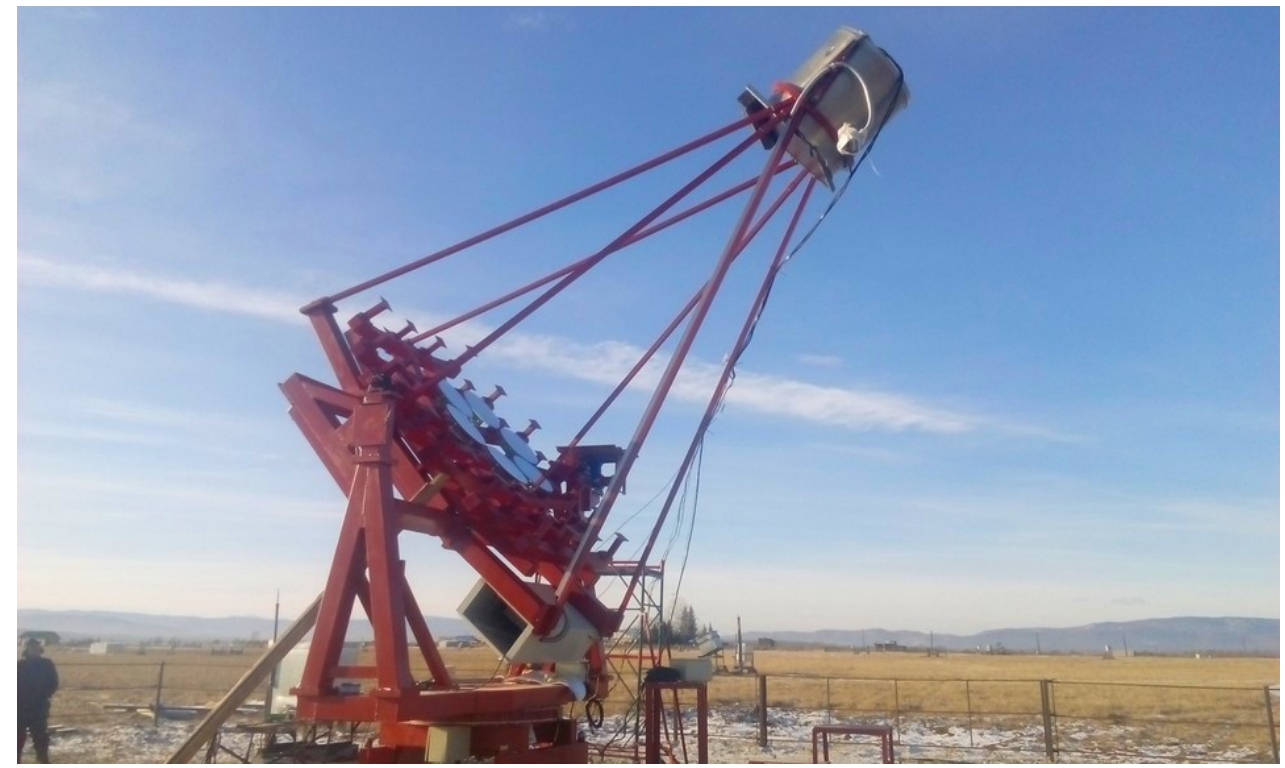

Figure 1: The first TAIGA-IACT

\section{Camera of the TAIGA-IACT}

\subsection{Structure}

The telescope has a composite reflector of Davis-Cotton design consisting of 34 spherical glass mirrors with $60 \mathrm{~cm}$ diameter each. The reflector has a total diameter of $4.3 \mathrm{~m}$. Its focal length is $4.75 \mathrm{~m}$.

The camera of TAIGA-IACT includes a matrix of photomultipliers (PMTs), high voltage system, signal processing and readout electronics and power supply controller. Construction of the camera provides its reliable operation in the cold winter conditions. That is achieved by use of a thermally insulated housing and temperature control system. The input window of the camera is made of plexiglass with a thickness of $1.5 \mathrm{~cm}$ and its surface is blown by the heated air that circulates inside the camera housing. Owing to such forced air circulation the 
temperature variation inside the camera housing does not exceed 2-3 degrees. To protect PMTs from day light the input window is remotely shut tight using ordinary blinds.
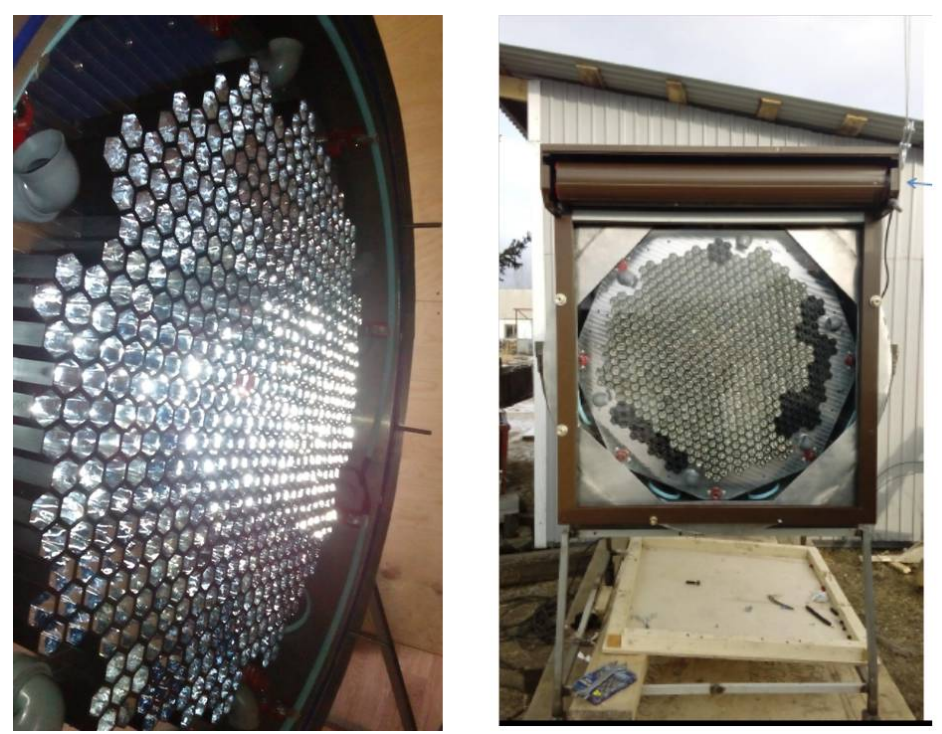

Figure 2: The TAIGA-IACT camera

The camera consists of 560 hexagonally packed PMTs (figure 2). PMTs are grouped into clusters. A single cluster includes 28 PMTs (4 groups of 7 PMTs) and the following electronics: a signal processing board based on MAROC3 chip, 4 high voltage divider boards, 4 high voltage power supplies, an auxiliary board with connectors, with DAC to control high voltage and with ADC to measure PMT anode currents (figure 3, left). High voltage divider board together with high voltage power supply are designed for group of 7 PMTs. Clusters are mounted on a duralumin plate with a thickness of $2 \mathrm{~cm}$. On the other side of the plate each PMT is equipped by Winston cone to increase sensitive area by a factor of $\sim 4$. Winston cones are designed in such a way that a single pixel of the camera views the whole reflector of the telescope. The camera provides a FoV of $\sim 10^{\circ}$. Angular size of a single pixel is $\sim 0.36^{\circ}$.
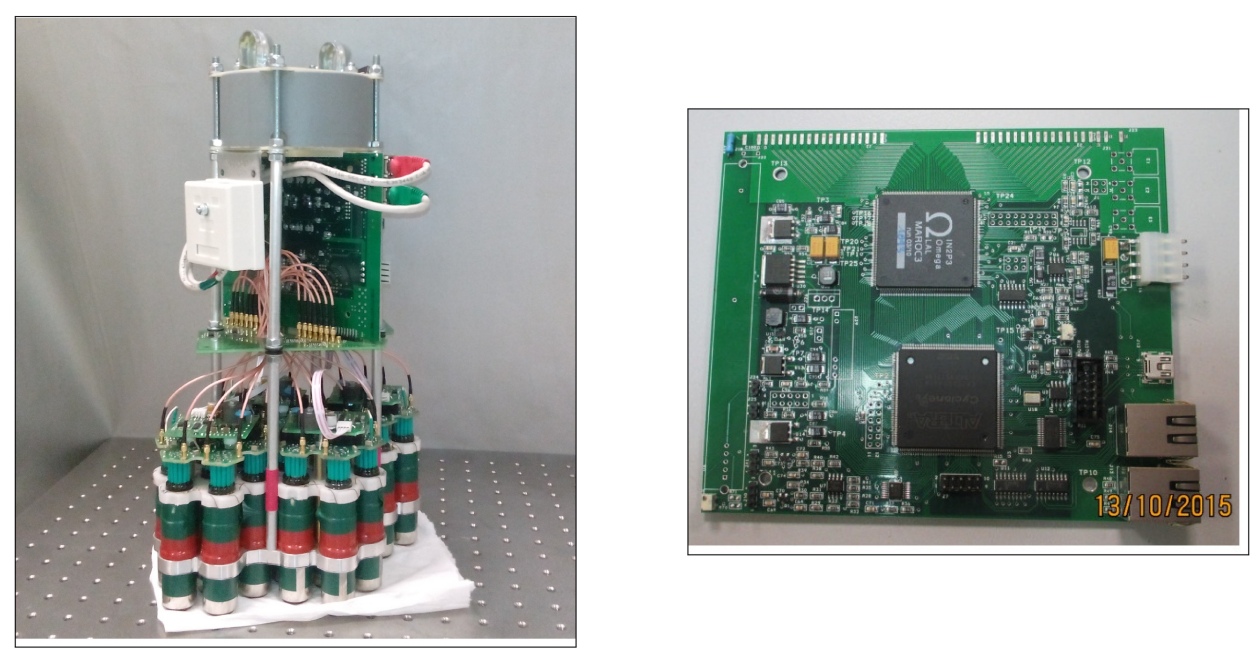

Figure 3: Cluster of 28 PMTs (left) and MAROC Board (right) 


\subsection{Camera electronics system}

The camera electronics system [5] can be divided into front-end, signal processing and readout electronics. Front-end electronics include PMTs XP1911 and high voltage system. PMT has short 8-dynode high voltage divider. PMT gain of $\sim 1 \cdot 10^{5}$ is limited by night sky background light.

The signal processing system consists of identical boards (MAROC Boards), each of them serves a single cluster. The MAROC Board provides detection of signals from 28 PMTs, monitoring of counting rates, DC anode currents and high voltage control.

The signal processing system is based on 64-channel chip ASIC MAROC3 (figure 3, right). In each MAROC channel PMT signal is amplified by a preamplifier with adjustable gain. Then the amplified signal is split into two parallel channels. The first channel provides a charge measurement by an integrating amplifier followed by a slow shaper. The peaking time corresponds to $35 \mathrm{~ns}$. The charge of the pulse is stored in a buffer and transferred to an analog multiplexed output that comes to external 12-bit serial ADC. In this channel signals measurement can also be performed by internal 12-bit Wilkinson ADC which has a digital multiplexed output.

The trigger output is produced using fast shaper and discriminator in the second channel. Discrimination threshold is set by internal 10-bit DAC. The trigger output signal is fed to the FPGA (EP1C6Q240C6). FPGA generates the trigger of a cluster according to the specified number of received trigger signals within 15-ns time interval.

Signals from each PMT come in parallel to two MAROC channels with preamplifiers with high and low gain that allows to extend dynamic range. All MAROC channels were calibrated using pulse generator.

The readout system consists of the central control board (Central Controller). The Central Controller manages the MAROC Boards, produces the common trigger according to the number of triggered pixels, provides data acquisition from the MAROC Boards, storing data in a buffer and data transmission to PC (figure 4, left). The imaging camera is synchronized to GPS time and with the TAIGA-HiSCORE array using the Central Controller.

The Central Controller is based on FPGA Xilinx Spartan-6 with a system frequency of 100 MHz. The local time clock of the Central Controller operates at the frequency of $200 \mathrm{MHz}$. The MAROC Boards can be connected to 24 interface channels of the Central Controller. Communication between the MAROC Boards and the Central Controller is made under LVDS standard and provides timing less than $5 \mathrm{~ns}$ and data transmission rate more than $20 \mathrm{Mbit} / \mathrm{s}$. Data exchange with the control PC is provided via the TCP/IP Ethernet interface with the rate of $100 \mathrm{Mb} / \mathrm{s}$.

The power supply controller (Power Controller) has twenty four +12 -volt channels for the MAROC Boards and the same number of +24 -volt channels for high voltage systems (figure 4, right). These channels are independently controlled by the Central Controller via RS-485 interface with baud rate of 115200 . The Power Controller was developed also on the basis of FPGA Xilinx Spartan-6. 

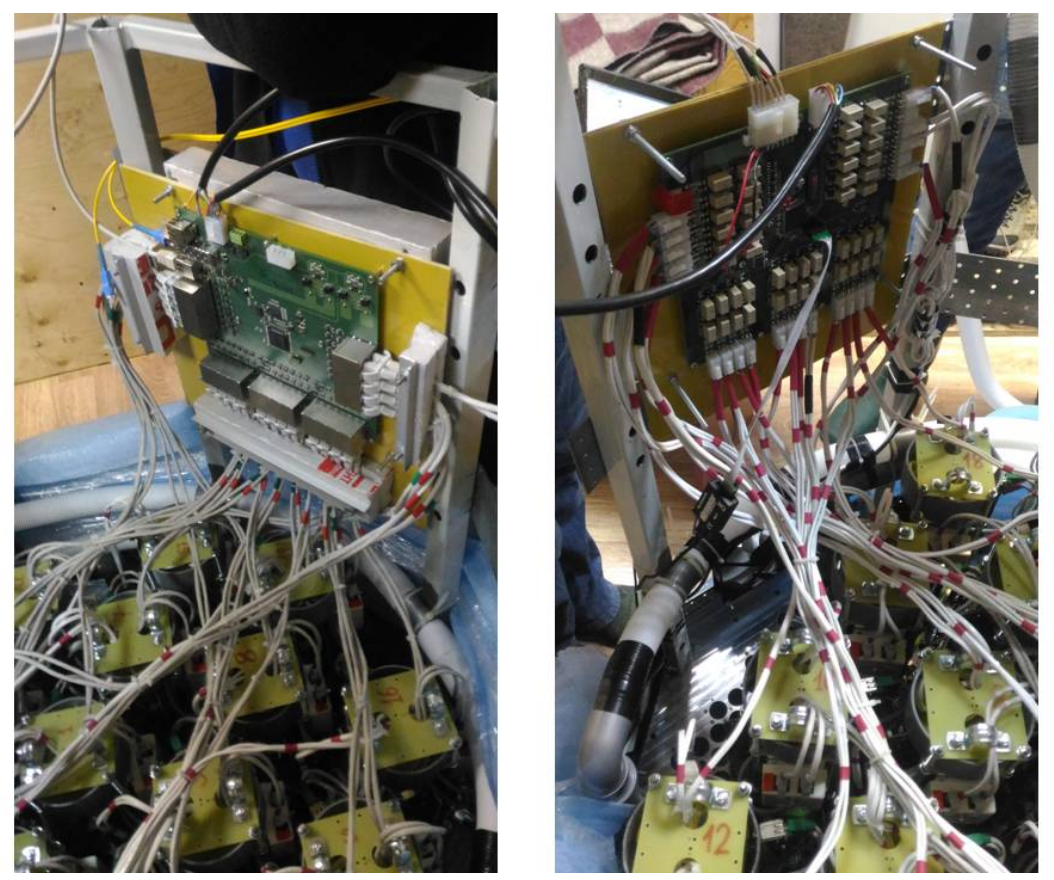

Figure 4: The Central Controller (left) and the Power Controller (right)

\section{Calibration of the TAIGA-IACT camera}

\subsection{Calibration light source}

To monitor PMT parameters and related electronics of the TAIGA-IACT camera a calibration pulsed light source based on LEDs is under development. Such a calibration system should have fast light emission kinetics of a few nanoseconds and a variable light yield in the wide dynamic range of up to 3000 p.e. per PMT. Also it would be desirable to have several LEDs emitting at different wavelengths matching PMTs photocathode spectral sensitivity.

The test calibration light source was developed for preliminary measurements. It consists of identical LED drivers developed especially for the Tunka-133 array [6, 7] and equipped with ultra bright BL-L513UBW blue LEDs with a diffusing lens. High reliability of these LED drivers has been proven by their successful operation in the Tunka-133 experiment for many years. Intensity of light pulses is controlled by changing of the driver's supply voltage in the range of $0-12 \mathrm{~V}$. Light emission kinetics of the calibration light source was measured by time correlated photon counting technique using fast PMT XP2020. Light pulse width (FWHM) is $\sim 3.3$ ns (figure 5). 


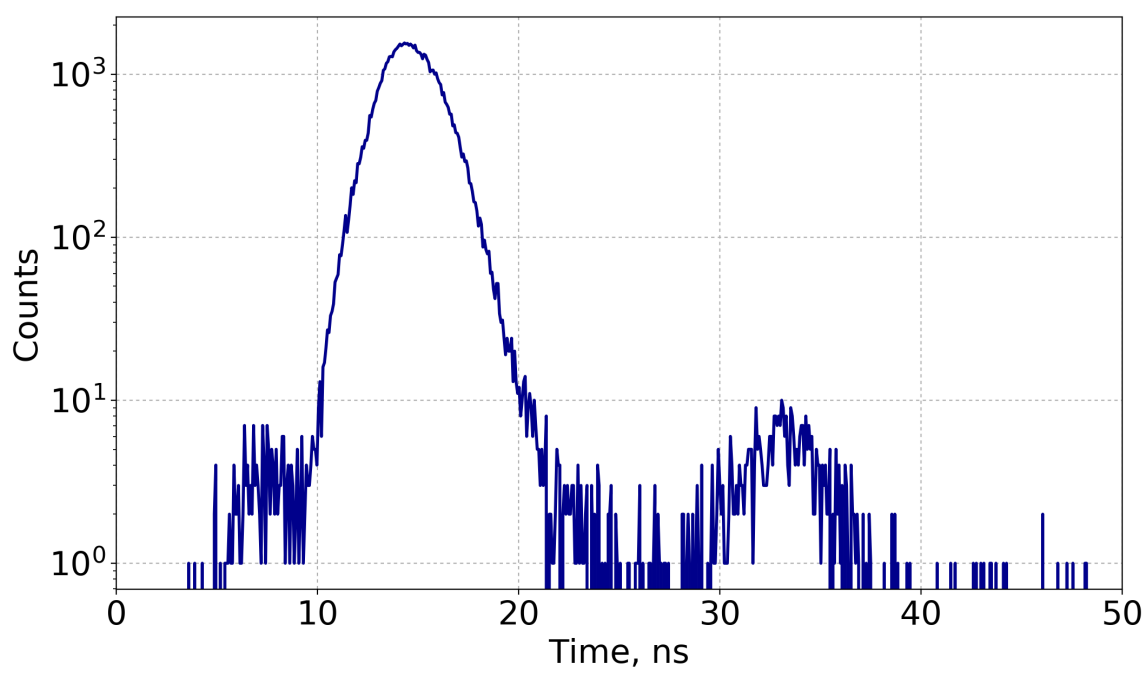

Figure 5: Light emission kinetics of the calibration light source

The calibration light source was mounted close to the center of the reflector and remotely controlled by 12-bit DAC and pulse generator. The nonuniformity of the camera illumination by this light source was carefully measured in the laboratory. The illumination intensity decreases smoothly towards the edge of the camera by less than $5 \%$.

\subsection{Linearity of readout chain}

The linearity of the whole readout chain consisting of PMT, MAROC channel and external ADC was measured using the calibration light source and a double illumination method (figure 6). MAROC channels with high gain are designed for detection of low intensity light pulses up to $\sim 150$-200 p.e. On the other hand low gain MAROC channels allow to increase dynamic range up to $\sim 3000-3500$ p.e.
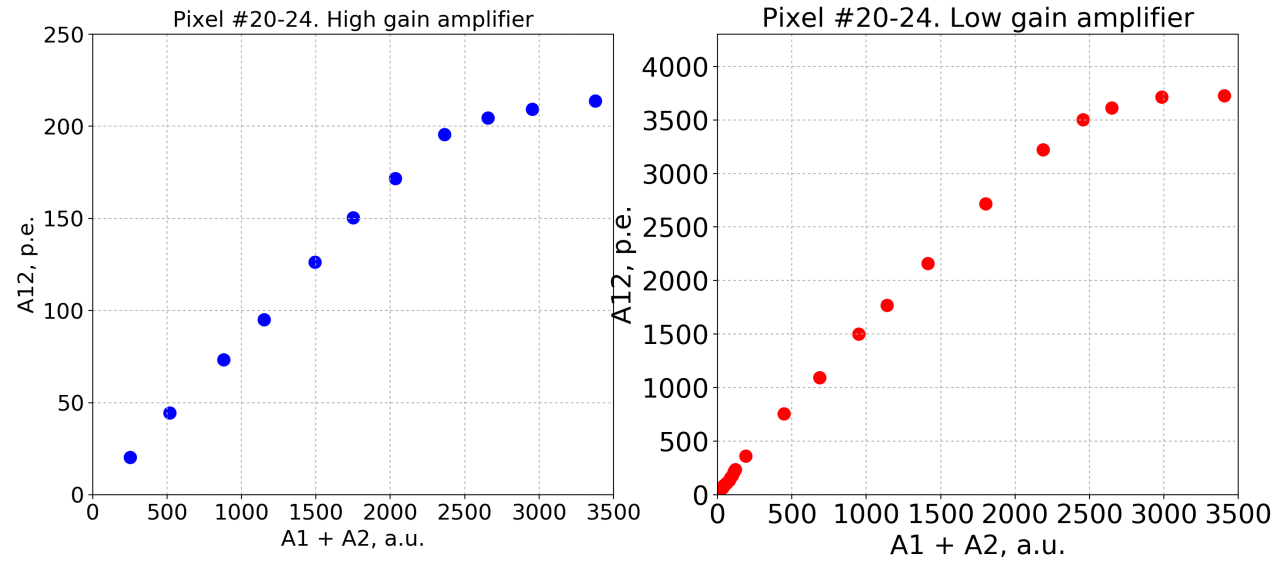

Figure 6: Linearity of MAROC channels with high (left) and low (right) gain amplifiers 


\subsection{Relative sensitivity of pixels}

Sensitivities of individual PMTs in the camera differ very much from each other. Therefore a calibration of the camera by a light source is important to know a relative sensitivity of pixels. Pixel sensitivity was evaluated by measuring an average number of photoelectrons registered in response to light pulses from the calibration source. The relative sensitivity of pixels can be obtained by comparing of the number of registered photoelectrons (figure 7). The number of photoelectrons was calculated using PMT gain previously measured in the laboratory, conversion factor of MAROC channel and mean amplitude registered by the pixel. This method does not take into account a probable instability of PMT gain under night sky background light. Another method to determine the number of photoelectrons using multiphotoelectron peak and PMT's excess noise factor analysis will be checked.

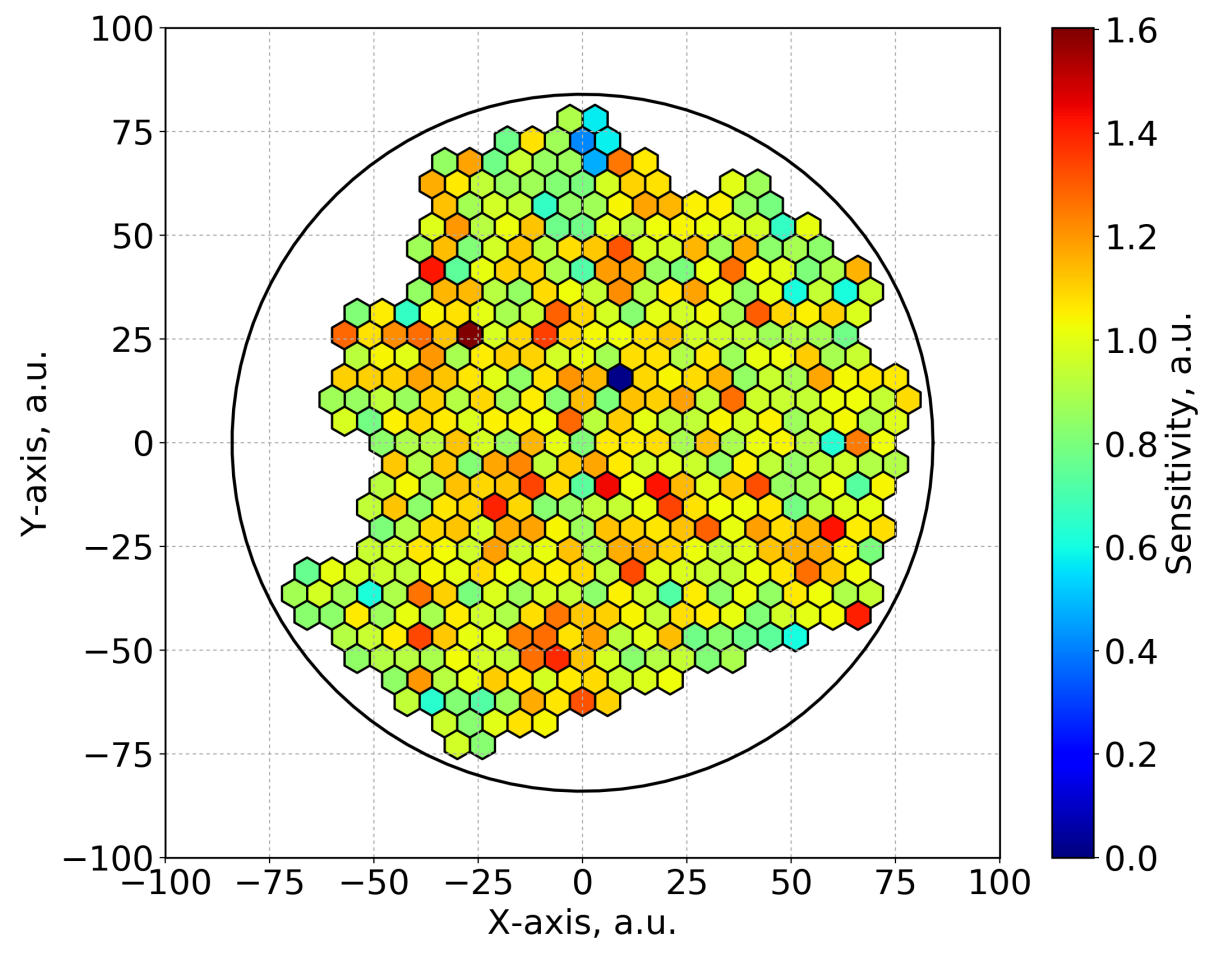

Figure 7: Relative sensitivity of the camera pixels (currently the camera is not completely filled with photomulipliers, the rest photomultipliers will be added in this summer) 


\section{Conclusion}

The first TAIGA-IACT was put in commission in December 2016. The imaging camera has 560 PMTs. The camera electronics on the basis of 64-channel chip ASIC MAROC3 provide detection of PMT signals over a wide dynamic range of up to 3000 p.e., monitoring of counting rates, DC anode currents and high voltage control.

The imaging camera of TAIGA-IACT needs a regular optical calibration to obtain accurate experimental data. The nanosecond LED-based calibration system with adjustable light yield was developed for this purpose. Using the calibration light source a relative sensitivity of pixels was measured as well as linearity of readout chain.

\section{Acknowledgement}

This work was supported by the Russian Foundation for Basic Research (grants 16-2913035) and the grant 15-12-20022 of the Russian Science Foundation (section 1 and 2).

\section{References}

[1] N. Budnev et al. (TAIGA Collaboration), The TAIGA experiment: From cosmic-ray to gamma-ray astronomy in the Tunka valley, Nucl. Instrum. and Meth. A. 2017. V.845, P.330

[2] N. Budnev et al. (TAIGA Collaboration), The TAIGA experiment - a hybrid detector for very high energy gamma-ray astronomy and cosmic ray physics in the Tunka valley. These conference proceedings

[3] L. Sveshnikova et al. (TAIGA Collaboration), Commissioning the joint operation of the wide angletiming HiSCORE Cherenkov array with the first IACT of the TAIGA experiment. These conference proceedings

[4] M. Tluczykont et al. (TAIGA Collaboration), TAIGA-HiSCORE: results from the first two operation seasons, these proceedings. These conference proceedings

[5] I. Yashin et al. (TAIGA Collaboration), Imaging Camera and Hardware of Tunka-IACT, PoS ICRC2015 (2016) 986

[6] B.K. Lubsandorzhiev et al., A LED Flasher for Tunka experiment, Proceedings of the 30th ICRC, Merida, Mexico, 2007

[7] S. Berezhnev et al., The Tunka-133 EAS Cherenkov light array: Status of 2011, Nucl. Instrum. and Meth. A. 2012. V.692, P.98 